\title{
UN MODÈLE POUR LE JAPON? NAKAE CHÔMIN ET LA CONSTITUTION BRÉSILIENNE DE 1824.
}

Eddy Dufourmont ${ }^{1}$

Summary: The article analyses the translation of the Brasilian Constitution of 1824, published in the journal headed by Nakae Chômin, Ôbei seiri sôdan. Setting this translation in its historical background and comparing it with the original French text shed light on the underlying political issue. While Japan was experiencing intense political struggles between the Democrats and the government, who wanted an authoritarian constitution centered on the emperor, the translation of the 1824 Constitution was a tool to promote a constitutional monarchy.

Keywords: Nakae Chômin, Brasilian Constitution of 1824, Meiji, translation, French republicanism, Benjamin Constant, Jean-Jacques Rousseau.

Résumé: L'article analyse la traduction de la Constitution brésilienne de 1824, parue dans la revue dirigée par Nakae Chômin, Ôbei seiri sôdan. Replacer cette traduction dans son contexte et la comparer avec le texte original français utilisé permet d'éclaircir l'enjeu politique qui lui était sous-jacent : à un moment où le Japon connaissait des luttes politiques intenses entre les démocrates et le gouvernement, qui désirait une constitution autoritaire centrée sur l'empereur, la traduction de la Constitution de 1824 permettait de promouvoir une monarchie constitutionnelle. Mots clés: Nakae Chômin, Constitution brésilienne de 1824, Meiji, traduction, républicanisme français, Benjamin Constant, Jean-Jacques Rousseau.

1 Maître de conférences HDR, Université Bordeaux Montaigne, France. 


\section{Introduction}

De longue date, l'histoire des relations entre le Japon et le continent sudaméricain, notamment avec le Brésil, a pu focaliser l'attention des recherches du fait de la présence ancienne d'émigrés japonais ${ }^{2}$. Mais nous souhaitons proposer ici une autre perspective, sur le plan de l'histoire politique et intellectuelle. S'il est bien connu que le Japon des premières décades de l'ère Meiji (1868-1912) s'est tourné essentiellement vers l'Europe de l'Ouest et les Etats-Unis pour bâtir un Etat-nation et une économie industrielle, la place du Brésil est quasi ignorée en la matière. Il est vrai que le Brésil ne semble réellement apparaître dans les publications japonaises qu'à partir de 1890: une recherche documentaire sur le site de la Bibliothèque de la Diète, la plus importante du Japon, donne des dizaines de titres à partir de cette date, mais beaucoup moins avant elle. L'un de ces ouvrages est une encyclopédie ne consacrant qu'un nombre restreint de pages au Brésil. Ce pays est qualifié d'«empire» (Burajiru teikoku ブラジル帝国), comme le Japon, et il est même décrit comme l'un des plus grands existant sur terre, pourvu d'un régime monarchique et de deux chambres, d'une grande solidité ${ }^{3}$. Les autres ouvrages sont soit des encyclopédies de géographie traduites soit des ouvrages sur les régimes politiques. Si ces derniers se limitent à de brèves descriptions des institutions ${ }^{4}$, ils témoignent néanmoins d'une curiosité large, qui s'inscrit dans le Mouvement pour les libertés et les droits du peuple (Jiyû minken undô 自由民権運動) des années 18741890, par lequel les Japonais réclamaient au gouvernement la mise en place d'un parlement ainsi qu'une Constitution, qui leur garantirait les libertés fondamentales.

Dans ce contexte, la traduction de la Constitution brésilienne de 1824 dans la revue Ôbei seiri sôdan 欧米政理叢談(Collection de propos sur l'art du gouvernement), en 1883, apparait d'autant plus singulière. Cette revue était dirigée par Nakae Chômin 中江兆民 (1847-1901), l'un des plus importantes figures intellectuelles de l'époque, tour à tour penseur, traducteur, journaliste et militant du Mouvement pour les libertés et les droits du peuple. Il traduisait dans cette revue ou faisait traduire par ses élèves de son Ecole des études françaises (Futsugakujuku 仏学塾) une grande variété de textes

2 Il serait fastidieux et inutile ici de recenser toutes les recherches sur les rapports entre le Japon et le Brésil mais le lecteur pourra consulter la synthèse suivante, BAXTER, James, HOSOKAWA, Shûhei, отА Junk ed., Cultural exchange between Brazil and Japan. Immigration, history and language, Kyôto, International Research center, 2009.

3 TOMOYAMA, Saburô 伴山三郎, Kinsei bankoku chishi 近世万国地誌, Hakubunkan 博文館, 1889, pp.376-380. Avant cet ouvrage, en 1875, la traduction d'une encyclopédie anglaise de géographie consacre deux pages au Brésil (Chikyû setsuryaku yakkai地球説略訳解, Tôkyô, Etô Kihei 江藤喜兵 衛, vol.4, pp.33-5).

4 MIBASHI, Satoru 三橋惇, Bankoku kokkai taiy $\hat{\boldsymbol{o}}$ 万国国会大要 (Présentation des parlements du monde), Tôkyô, Gekigekidô 嚴々堂, 1880, pp.58-61 ; MARTIN, Frederik, ISHIKAWA, Masami 石 川正美, trad., Manpô seitai ruikan 万邦政体類鑑 (Panorama des régimes politiques du monde [The Statesman's yearbook]), Tôkyô, Wada Atsurô 和田篤太郎, 1883, pp.120-5; KELTIE, James Scott, traducteur inconnu, Ôbei kakukoku kokkai kiyô 欧米各国国会紀要 (Précis des parlements d'Europe et d'Amérique [The Statesman's yearbook]), Tôkyô, Furukawa Seiken 古川誠顕, 1888, pp.169-175. 
francophones. C'est à partir de l'ouvrage du juriste Edouard Laferrière, Les Constitutions d'Europe et d'Amérique, que Chômin fait publier sur plusieurs numéros de Ôbei seiri sôdan la traduction de la Constitution de $1824^{5}$. Pourquoi celui qui a traduit Rousseau et fait connaître à ses contemporains les républicains français a-t-il choisi celle-ci en particulier et que signifie ce choix? Telles sont les questions que nous aborderons ici.

\section{Pourquoi le Brésil ? Nakae Chômin et la quête de la démocratie}

Si le rôle des intellectuels-traducteurs dans la transformation du Japon dans les années 1870-1880 est bien connu, Nakae Chômin occupe une place à part : il est resté dans les mémoires, à juste titre, pour sa traduction du Contrat social de Jean-Jacques Rousseau et ses liens avec la France, où il fut l'un des rares à y séjourner pour études, entre 1872 et 1874. Mais cette image du «Rousseau d'aujourd'hui» (Kon Rusô 今蘆 騒) ou encore de «Rousseau de l'Orient» (Tôyô no Rusô 東洋のルソー), telle qu'elle s'établit de son vivant ${ }^{6}{ }^{\prime}$ 'est que partiellement vraie car incomplète, pour deux raisons.

Tout d'abord, Rousseau ne doit pas en effet occulter une œuvre de traduction bien plus grande qu'on l'imaginait: Chômin a fait traduire également plusieurs républicains français sous forme de livres et dans sa revue Ôbei seiri sôdan, dont le contenu était exclusivement des traductions de textes français portant sur des sujets très divers, de la philosophie à la politique, en passant par le droit, l'économie et la philosophie? D'autre part, Chômin a eu un regard global qui ne s'est pas focalisé exclusivement sur la France. Il est vrai que la France est très souvent citée comme modèle, mais aux côtés de l'Angleterre. Constamment associés comme modèles de pays civilisés dans ses articles, leurs hommes politiques sont aussi cités en exemple par Chômin ${ }^{8}$. Ils jouent un rôle complémentaire, qui se résume bien dans ses Dialogues politiques entre trois ivrognes: «les Anglais sont intelligents, les Français passionnés. Les Anglais sont calmes, les Français impétueux. Une fois qu'ils ont emprunté le chemin du progrès, les Anglais ne se perdent plus en route. Les Français sont prompts à avancer, mais ils reculent tout

5 Comme il est spécifié dans chaque numéro de Ôbei seiri sôdan, Chômin se réservait un droit de regard (etsuran 閲覧) sur toutes les traductions. Celle de la constitution brésilienne de 1824 est d'auteur inconnu mais il ne fait donc guère de doutes que Chômin l'examina avant publication.

6 IDA Shin.ya 井田進也 et MATSUNAGA, Shôzô松永昌三, «Kaidai» 解題 (Commentaire), dans Nakae Chômin zenshî 中江兆民全集 (ci-après abrégé en NCZ), Tôkyô, Iwanami shoten岩波書店, vol.1, p.294.

7 Si Ida Shin.ya a fait œuvre de pionnier en mettant en avant l'existence de la revue Ôbei seiri sôdan, nous avons été le premier à en faire une étude exhaustive pour notre habilitation à diriger des recherches, dont le premier article est en partie issu. Voir DUFOURMONT, Eddy, Un cas d'étude de transfert culturel au Japon : Nakae Chômin (1847-1901), Rousseau et le républicanisme français, mémoire soutenu le 18 novembre 2016 à l'EHESS, sous la direction d'Anne Cheng.

8 NAKAE, Chômin, «Sakuron» 策論 (Plan de mesures, 1875), NCZ, 1, p.27; Chômin, Senkyonin no mezamashi 選挙人の目覚まし (Le Réveil de l'électeur, 1890), NCZ, 10, pp.98-119. 
aussi vite» ${ }^{9}$. En fait, il est même permis de dire que l'Angleterre jouit chez Chômin d'une image aussi positive que la France, sinon plus: dans Ôbei seiri sôdan, même si la France a le crédit d'avoir été plus novatrice dans sa révolution de 1789 en comparaison de celle de $1688^{10}$, dans son ouvrage sur la Révolution française, Chômin critique la Terreur pour la violence dont elle a fait preuve ${ }^{11}$. Dans un article de 1888, le ton devient plus critique et sévère : les Français sont décrits comme des «chiens fous», des instables dominés par leurs sentiments. Cette maladie leur fait succéder les Premiers ministres et les rend facilement séduits par les discours grandiloquents des deux Napoléon et aujourd'hui du général Boulanger ${ }^{12}$. Des Anglais pacifiques et des Français violents: Chômin ne suggérait-t-il pas ainsi aux Japonais de se tenir entre les deux, de concilier la modération britannique et la passion démocratique française?

Mais Chômin n'a pas été sans ignorer les autres pays, par exemple ceux de la Péninsule ibérique. Ainsi Chômin écrit au sujet de l'Espagne que celle-ci est «tombée au deuxième rang» et qu' «elle a besoin de routes, d'éducation et de démantèlement de la domination religieuse» ${ }^{13}$. À l'inverse, Chômin fait grand cas de petits pays qui malgré leur taille ont su protégé leur indépendance, tels que la Suisse, la Belgique et la Hollande $^{14}$. Pour Chômin la Suisse, «bien que plus petite que l'Allemagne, la France et l'Angleterre est aussi développée qu'eux et que si elle est aussi riche elle l'est surpasse par la beauté de ses mœurs $(. ..){ }^{15}$. On le voit, Chômin s'est servi des différents pays comme modèle ou contre-modèle. De l'ensemble il se dégage nettement l'idéal d'un pays qui arrive à se bâtir comme puissance économique et culturelle, en se défaisant des entraves issues de la féodalité, y compris celles de la religion. En fait Chômin avait à cœur que son pays se modernise et fasse partie des démocraties en même temps que des grandes puissances économiques et cette attitude transparaît dès son premier texte de $1874^{16}$. Il n'est donc guère étonnant que son attention ait pu se fixer, même ponctuellement, sur le Brésil et sa constitution.

La traduction de la Constitution brésilienne a occupé plusieurs numéros de

9 NAKAE, Chômin, Dialogues politiques entre trois ivrognes, traduit par Eddy Dufourmont et Christine Lévy, CNRS, Editions, 2008, p.70.

10 FOUCHER DE CAREIL, Louis, «Révolution d'Angleterre de 1688», dans Maurice Block, Dictionnaire général de la politique, O.Lorenz, 1873-4, tome 2, pp.849-851.

11 NAKAE, Chômin, Furansu kakumei niseiki kiji 革命前法蘭西二世紀事 (Récit des deux siècles de la France avant sa révolution, 1886), NCZ, 8, p.148.

12 NAKAE, Chômin, «Bûlanjê shôgun» ブーランジェー 将軍 (Le général Boulanger, 7 décembre 1888), NCZ, 14, pp.310-3.

13 NAKAE, Chômin, «Supein» 西班牙国 (L’Espagne, 1888), NCZ, 11, p.307.

14 NAKAE, Chômin, «Rongaikô» (Sur la politique étrangère, 12, 15, 17 août 1882), NCZ, 14, p.136.

15 NAKAE, Chômin, «Suisu» 端西国 (La Suisse, 1888), NCZ, 11, p.325-8.

16 NAKAE, Chômin, «Sakuron», op.cit., p.30. 
Ôbei seiri sôdan mais une première remarque s'impose: outre cette traduction Ôbei seiri sôdan propose également celle de la constitution d'Angleterre et de Belgique, à partir d'un ouvrage d'Edouard Laferrière, Les Constitutions d'Europe et d'Amérique, pour le Royaume-Uni et le Brési1 ${ }^{17}$, et La Constitution belge annotée, de Jean-Joseph Thonissen $^{18}$. Si la place importante de l'Angleterre chez Chômin explique aisément son choix, celui de la Belgique et du Brésil offre une particularité: dans le cas de la Belgique, il s'agit de la Constitution de 1831, qui marque l'indépendance de la Belgique vis-à-vis des Pays-Bas et dans le cas du Brésil il s'agit de la Constitution de $1824^{19}$, qui marque elle aussi l'indépendance du pays vis-à-vis du Portugal. Autrement dit le Brésil et la Belgique étaient des pays neufs, situation qui a pu apparaître, aux yeux de Chômin, proche de celle du Japon, pays alors lui aussi en construction. La traduction présente d'ailleurs l'année 1824 comme celle de la «fondation du pays» (kenkoku 建国) là où il est question d' «indépendance» dans le texte original. Le terme « constitution» lui-même est traduit parfois par «loi de fondation du pays» (kenkokuhô 建国法) ${ }^{20}$. La différence au niveau sémantique est minime mais la nuance est importante puisqu'en choisissant kenkoku plutôt que dokuritsu (le terme japonais pour indépendance) la traduction suggère une similitude entre le Japon et le Brésil. Le parallèle pouvait d'autant plus être manifeste pour les traducteurs que le Brésil comme le Japon étaient gouvernés par des empereurs. Le traducteur ne se prive d'ailleurs pas d'utiliser le terme kôtei 皇帝au sujet de l'empereur du Brésil, normalement utilisé pour le souverain japonais ${ }^{21}$.

Si d'autres pays auraient pu être choisis sur ce point, il y a une autre similitude entre l'Angleterre, la Belgique et le Brésil qui explique le choix de ces pays et pas d'autres: les constitutions traduites établissaient des monarchies constitutionnelles représentatives. Pour un Japon dominé alors par un empereur mais sans rôle encore bien défini, faute de constitution, ce choix semble très parlant: Chômin semble avoir voulu trouver dans l'Angleterre, la Belgique et le Brésil des sources d'inspiration pour bâtir une monarchie constitutionnelle. L'attirance de Chômin pour la France de 1789 laisse souvent penser qu'il était partisan de la république mais la réalité est plus complexe: dans son article bien connu de 1881, «Sur l'association du souverain et du peuple dans le gouvernement», Chômin montre que la république n'est pas forcément

17 L'ouvrage offre également les traductions des constitutions égyptienne et ottomane.

18 LAFERRIÈRE, Edouard, Les Constitutions d'Europe et d'Amérique, Paris, Cotillon, 1869, Brésil. Constitution du 25 mars 1824 avec les modifications et additions résultant de la loi des réformes constitutionnelles du 12 août 1824, pp.589-597. «Burajîru kokken»巴西ブレジール国権 (Constitution du Brésil), Ôbei seiri sôdan, 36, 38, 40, 43, 49, 52, 55.

19 Le principal auteur de la constitution est José Joaquim Carneiro de Campos (1768-1836).

20 Ôbei seiri sôdan, 52, p.1837.

21 LAFERRIÈRE, op.cit., p.590; Ôbei seiri sôdan, 36, p.1289. 
synonyme de démocratie et qu'une monarchie n'est pas nécessairement une tyrannie ${ }^{22}$. Chômin ne prenait pas explicitement position pour la monarchie constitutionnelle mais par la suite il insista sur la possibilité de combiner monarchie et démocratie: en 1887, Chômin explique que dans un gouvernement constitutionnel sain le premier ministre procède toujours des décisions du Parlement et que le souverain à la tête du pays doit être respecté mais, comme les démons et les dieux ${ }^{23}$, doit être complètement inactif et en dehors des vagues de la politique, autrement dit irresponsable ${ }^{24}$. Chômin utilise le discours officiel qui fait de l'empereur un dieu pour lui enlever toute possibilité d'agir en autocrate (au contraire de ce que feront les oligarques avec la Constitution de 1889). La traduction de la constitution brésilienne, ainsi que celle de Belgique et d'Angleterre, semble abonder dans ce sens. Ida Shin.ya ajoute à ce sujet qu'en août 1882, pendant le voyage en Allemagne d'Itô Hirobumi 伊藤博文, le principal oligarque, le secrétaire du Genrô.in Kaneko Kentarô 金子堅太郎 fit l'éloge de la constitution brésilienne, arguant qu'elle résultait de l'étude des autres pays. Il essayait là de camoufler l'objectif d'Itô qui était de prendre modèle le régime autoritaire prussien pour élaborer la constitution japonaise ${ }^{25}$.

Il faut relever ici que parmi les sources francophones de Chômin figurent le Dictionnaire général de la politique, dirigé par Maurice Block, dont un certain nombre d'articles furent traduits dans Ôbei seiri sôdan ${ }^{26}$. Un article est consacré au Brésil. S'il est difficile d'affirmer que Chômin l'a réellement lu, on peut toutefois indiquer que l'article présente lui aussi ce pays comme un terre immense et sous-peuplée, partagée entre plusieurs «races». Surtout il détaille le contexte historique dans lequel la Constitution de 1824 fut promulguée, et y souligne la place de Benjamin Constant, «mieux inspiré que Rousseau, qui, dominé par ses instincts socialistes, n'offrait aux peuples que des constitutions impossibles ${ }^{27}$. L'article décrit ainsi l'empereur comme un souverain régnant mais ne gouvernant pas, et il caractérise la constitution par la

22 NAKAE, Chômin, «Sur l'association du peuple et du monarque dans le gouvernement» (Kunmin kyôji no setsu 君民共治の説 ; 24 mars 1881), NCZ, 14, pp.10-12.

23 Allusion aux Entretiens de Confucius, VI, 22, p.59: «Honorer esprits et démons tout en les tenant à distance».

24 NAKAE, Chômin, «Tatô no kaibutsu» 多頭の怪物 (Le monstre polycéphale; 27 décembre 1887), NCZ, 11, p.45.

25 IDA Shin.ya, Nakae Chômin no Furansu 中江兆民のフランス (La France de Nakae Chômin), Tôkyô, Iwanami shoten, 1987, p.70.

26 BLOCK, Maurice ed., Dictionnaire général de la politique, op.cit. Articles traduits: Clément Ambroise, «impôts»; Read Charles, «Homme d'Etat»; Mazade Louis «Institutions politiques»; Foucher de Careil Louis «Révolution d'Angleterre de 1688»; Block Maurice, «Partis», «Club», Simon Jules «Ambition»; Chédieu Emile «Despotisme» et «Opposition», Helie Faustin «Guerre civile»; Lavollée Charles-Hubert «Homme politique, «Publiciste»; Herve Edouard «Leaders».

27 REYBAUD, Charles, «Brésil», Dictionnaire général de la politique, op.cit., tome 1, p.256. 
primauté du parlement sur l'empereur, l'existence des assemblées régionales ayant pouvoir législatif.

À vrai dire, la traduction de la constitution belge diffère des deux autres car elle se limite à deux passages du commentaire de l'article 25 affirmant la souveraineté de la nation. La traduction prend d'ailleurs le titre de «Commentaire sur la souveraineté» (shuken no kai 主権)解). La traduction de la Constitution brésilienne est, elle, complète. Il faut souligner que la version choisie pour la traduction inclut l'amendement décentralisateur du 12 août 1834. La Constitution de 1824 est une sorte de compromis entre libéralisme et absolutisme, constitutionalisant l'absolutisme via l'introduction du «pouvoir modérateur» (nous y reviendrons) et l'amendement lui-même allant dans le sens du libéralisme en autorisant la création de chambres délibératives provinciales, avec liberté pour elles de prélever les impôts et de disposer de leur propre corps de fonctionnaires, sous le commandement d'un directeur nommé par le pouvoir central ${ }^{28}$. Pour les partisans du Mouvement pour les libertés et les droits du peuple, qui se faisaient les défenseurs des paysans, au cœur des violences politiques de l'époque ${ }^{29}$, la Constitution brésilienne pouvait servir d'exemple.

\section{La traduction de la Constitution de $\mathbf{1 8 2 4}$}

Relevons tout d'abord que la traduction aurait dû être complète. Il manque seulement les titres VII (De l'administration des provinces) et VIII (Dispositions générales et garantie des droits civils et politiques des citoyens brésiliens) et le numéro qui publie la dernière traduction du texte indique bien à la fin la mention «inachevé» (mikan 未完) $^{30}$. Or ce numéro fut, contre la volonté de ses rédacteurs, le dernier de la revue. Si d'autres numéros avaient suivis, le traducteur aurait sans doute achevé son travail.

Les titres traduits constituent autant de thèmes centraux pour les partisans du Mouvement des libertés et des droits du peuple, qui plus est dans un ordre en apparence favorable aux droits du peuple: les citoyens (II), la représentation nationale (III), le pouvoir législatif (IV), le pouvoir exécutif et modérateur, à savoir l'empereur ainsi que les ministres (V), le pouvoir judiciaire (VI). L'empereur et les ministres viennent après les citoyens et le pouvoir législatif, avec beaucoup moins d'articles concernant les seconds que les premiers (52 contre 91).

28 BONAVIDES, Paulo, DE ANDRADE, Paes, História constitucional do Brasil, Brasília, Paz e Terra, 1991, pp. 95 sqq.

29 La majeure partie des partisans du Mouvement pour les libertés et les droits du peuple étaient des paysans, qui entendaient lutter contre des impôts trop élevés et contre les abus des administrations. De multiples révoltes paysannes égrénèrent les années 1880 , culminant avec la révolte de Chichibu en 1884, durant laquelle les paysans tentèrent de fonder une communauté autonome et marchèrent contre la capitale.

30 Ôbei seiri sôdan, 55, p.1886. 
La traduction débute par une introduction du traducteur, non identifié, ne figurant pas dans le texte original, comme cela arrive souvent dans la revue Ôbei seiri sôdan et dans les traductions de Chômin d'une manière plus générale. Elle présente le Brésil comme un pays riche du continent Sud-Américain, et explique que le pays a pris son indépendance en 1822 tout en gardant un souverain dans la famille royale portugaise. S'ajoutent à cela des affirmations révélatrices de l'intention du traducteur. Tout d'abord, celui-ci écrit qu' «un pays sans constitution [...] ne peut être appelé pays civilisé et doit être considéré comme barbare». Il ajoute que la Constitution brésilienne a la vertu de «protéger le prestige de l'Etat et de respecter la liberté du peuple (minjin no jiŷu 民人) 自由) $)^{31}$. La traduction utilise d'ailleurs le mot «peuple» (min 民) là où il est question de nation dans le texte original. Venant d'un membre du Mouvement pour les libertés et les droits du peuple, la nuance n'est pas négligeable. Par ailleurs, min est également utilisé pour traduire «citoyen», thème constituant le titre II de la constitution. On retrouve là une démarche similaire dans la traduction du Contrat social par Chômin (Min.yaku yakkai), dans un but de simplification pour le lecteur. La traduction utilise également les mêmes mots pour désigner les institutions que dans le Japon des années 1870-1880: l' «Assemblée générale 》 du texte original devient le «parlement» (kokkai 国会), le Sénat est traduit par Genrô.in 元老院 ${ }^{32}$ et la chambre des députés par Taigi.in 代議院 ${ }^{33}$.

Ces deux affirmations constituent précisément les idées directrices des partisans du Mouvement pour les libertés et les droits du peuple. Revenons à la première, qui fait d'un pays doté d'une constitution un pays civilisé. Le thème de la civilisation (bunmei 文 明) était dominant dans les discours de l'époque, qui, d'une manière unanime, appelait le Japon à faire partie des «pays civilisés». Fukuzawa Yukichi 福沢諭吉 (1837-1901) joua un rôle central dans l'introduction de cette notion avec la publication en 1875 d'une Ebauche de la théorie de la civilisation (Bunmeiron no gairyaku 文明論之概略). Cet ouvrage s'inspira beaucoup de l'Histoire de la civilisation en Europe de François Guizot (1828) ainsi que l'Histoire de la civilisation en Angleterre de Thomas Henry Buckle (1872-3). C'est autour de Fukuzawa que les premiers intellectuels formés à l'Europe constituèrent la Société de l'an 6 de Meiji pour appeler les Japonais à «s'ouvrir à la civilisation» (bunmei kaika 文明開化). Cet appel fut entendu puisque ce slogan devint le terme à la mode ${ }^{34}$. Chômin s'inscrit d'autant plus dans cet intérêt pour la

31 Ôbei seiri sôdan, 36, 1883, p.1286.

32 Le Genrô.in, «Chambre des Anciens» ou «Sénat» était le nom d'une institution fondée en 1875, lors d'un rapprochement temporaire entre Itagaki Taisuke 板垣退助, meneur du Mouvement pour les libertés et les droits du peuple, et les oligarques. Elle était chargée de mener un travail d'élaboration de la constitution mais se retrouva vite paralysée lorsqu'Itagaki se rendit compte que les oligarques ne comptaient absolument pas instaurer des libertés et la quitta.

33 LAFERRIÈRE, op.cit., p.590; Ôbei seiri sôdan, 36, p.1290 ; 38, p.1387.

34 OGAWARA, Masamichi 小川原正道, Fukuzawa Yukichi no seiji shisô 福澤諭吉の政治思想 (La Pensée politique de Fukuzawa Yukichi), Tôkyô, Keiô gijuku daigaku shuppankai 慶應義塾大学出版 会, 2012, pp.89sqq. 
civilisation que Guizot et Buckle sont précisément des auteurs traduits dans Ôbei seiri sôdan, surtout Guizot ${ }^{35}$. Or si en se faisant historien et en soumettant l'histoire à la civilisation, Guizot entendait donner une identité et une mémoire à la bourgeoisie (« les classes moyennes »), qui devenait acteur de l'histoire ${ }^{36}$, il est remarquable que dans les passages choisis de Guizot, Chômin et ses disciples ont systématiquement remplacé la bourgeoisie et les classes moyennes par le mot «peuple». Ils ont donc repris la vision de l'histoire de Guizot mais l'ont détourné.

Cette démarche peut être reliée à l'affirmation selon laquelle la constitution protège l'Etat et respecte la liberté du peuple. En liant constitution et civilisation, Chômin cherche habilement à donner des arguments imparables face au gouvernement qui est était prompt à faire sien l'appel à la civilisation mais l'était beaucoup au sujet de la constitution. L'argument implicite de l'introduction n'est d'ailleurs pas différent du fameux Mémorandum de 1874, l'un des textes qui lança le Mouvement pour les libertés et les droits du peuple: les auteurs justifiaient déjà l'établissement d'un parlement et d'une constitution en regard des progrès de la science ${ }^{37}$. Ainsi, en prenant le Brésil comme exemple de pays doté d'une constitution et civilisé, le traducteur suggérait que le Japon fasse de même pour lui aussi partie des pays civilisés. Il trouvait dans ce pays étranger un appui supplémentaire pour étayer l'argument classique liant constitution et civilisation.

Le deuxième argument pour justifier le choix de traduire la constitution brésilienne, l'alliance du prestige de l'Etat aux libertés du peuple, renvoie, lui aussi, aux débats en cours dans le Japon des années 1870-1880. En effet l' «Etat» (kakoku 家国) et le peuple dont il est question en préambule peuvent être reliés à l'article 3 où il est dit que l'Etat (seifu 政府 ${ }^{38}$ du Brésil est une monarchie héréditaire mais que son régime (seitai 政体) est constitutionnel et représentatif (rikken daigisei 立憲代議政) ${ }^{39}$. Or dans le Japon des années 1870 certains partisans et adversaires des droits du peuple mettaient, au départ, en opposition l'Etat (sous-entendu le souverain) et peuple, kokken (droits de l'Etat/de l'empereur) aux minken (droits du peuple). Mais, à partir de 1878, avec les tentatives de renégociation des traités inégaux il devint courant chez les défenseurs des droits du peuple, notamment Ueki Emori et Fukumoto Nichinan d'affirmer que la liberté

35 Chômin le fit traduire par Sakai Yûzaburô et Shiraishi Tokiyasu sous le titre Seiri shinron 政理新論 (Nouvelle théorie du principe politique), chez Nisshinkaku日新閣, en 1884.

36 ROSANVALLON Pierre, Le Moment Guizot, Paris, Gallimard, 1985, pp.193-203.

37 Voir la traduction dans DUFOURMONT, Eddy, Histoire politique du Japon, de 1853 à nos jours, Pessac, Presses Universitaires de Bordeaux, 2016, pp.74-6.

38 Le lexique moderne n'était pas encore fixé à l'époque et il était courant que des auteurs utilisent des mots disparus depuis ou bien utilisent des termes pourvus d'un sens différent par la suite, voire plusieurs termes pour le même mot. Tel est le cas ici puisque seifu désigne aujourd'hui «gouvernement» et kakoku est devenu kokka pour désigner l'Etat. 
du peuple contribuerait à renforcer la puissance du pays, en d'autres termes que minken serait utile à kokken. Les deux termes n'étaient plus mis en opposition. Quelques années plus tard, les fondateurs du Parti progressiste (Kaishintô 改進党) tenaient un discours similaire puisqu'ils réclamaient une monarchie à l'anglaise, un régime alliant un roi mais avec une constitution démocratique. Chômin lui-même prit la défense de cette option. Ainsi, la traduction de la constitution du Brésil servait d'argument supplémentaire à une revendication devenue commune au sein du Mouvement pour les libertés et les droits du peuple.

Il est remarquable que dans la Constitution brésilienne le lieu de la souveraineté ne soit pas clairement exprimé: si le souverain est le chef du pouvoir exécutif (article 102) et le détenteur du pouvoir modérateur (article 98, sesseiken 節制権), le parlement reçoit son serment. Ainsi, pour les traducteurs, la Constitution brésilienne pouvait permettre d'éviter le débat sur la souveraineté, qui avait déchiré les intellectuels japonais en $1881^{40}$, tout en affirmant implicitement la souveraineté du peuple, puisqu'à travers le parlement celui-ci détient la réalité du pouvoir, du moins une prépondérance. Le parallèle avec le Japon est d'autant plus évident que l'article 99 représente pratiquement à lui seul une anticipation de la constitution de 1889. En effet l'article dit :

«La personne de l'empereur est inviolable et sacrée. Elle n'est soumise à aucune responsabilité».

Ce que la traduction japonaise suit:

皇帝ノ身体ハ神聖ニシテ侵ス可ラズ故ニ皇帝如何ナル責任ヨモ有セズ ${ }^{41}$.

«Le corps de l'empereur est divin, saint et inviolable. Par conséquent l'empereur ne peut endosser aucune responsabilité»».

Comparons avec l'article 3 de la constitution de 1889:

天皇ハ神聖ニシテ侵スヘカラス.

«La personne de l'empereur est sacrée et inviolable».

40 Le Tôkyô nichinichi shinbun 東京日日新聞, proche du gouvernement, réagit à un débat suscité avec le Mainichi shinbun 毎日新聞au sujet de la souveraineté, entre octobre 1881 et mai 1882. Le premier défendait l'idée que la souveraineté réside chez l'empereur parce que celui-ci représentait une particularité unique au Japon. Un «contrat d’État» (kokuyaku 国約) et non un contrat du peuple devait être à la base de la constitution, ce qui était une manière d'affirmer la supériorité de l'État et de ses dirigeants, les oligarques, sur le peuple. Le second défendait l'idée que la souveraineté résidait dans le parlement et s'attachait à distinguer le monarque du Souverain.

41 LAFERRIÈRE, op.cit., p.604; Ôbei seiri sôdan, 52, p.1831. 
Cette apparente ressemblance ne fait qu'accentuer le fossé qui sépare l'empereur japonais et l'empereur brésilien. De par sa nature divine, l'empereur japonais aura en 1889 tous les pouvoirs et concèdera une constitution à ses sujets. L'empereur brésilien, lui, est un représentant du peuple (kokumin no dairisha 国民ノ代理者 dans la traduction), ce que ne sera jamais son homologue japonais. De par sa nature divine il n'aura aucun pouvoir si ce n'est le pouvoir modérateur, pouvoir d'indépendance, d'équilibre et d'harmonie entre les trois autres. Autrement dit, par un choix lexical, les traducteurs japonais semblent avoir délibérément suggéré la ressemblance entre l'empereur brésilien et l'empereur du Japon afin de détourner le discours sur sa divinité, dans un sens plus démocratique. Chômin fit d'ailleurs traduire dans Ôbei seiri sôdan deux textes affirmant la limitation nécessaire du pouvoir royal: 1'Essai sur le despotisme de Mirabeau ${ }^{42}$, qui constitue une mise en garde au roi contre la possible violence du peuple pour défendre sa liberté, et De l'organisation judiciaire et de la codification de Jeremy Bentham, où celui-ci cherche à définir le juge non pas comme une fonction autoritaire, au service d'une tyrannie, mais au contraire au service du peuple ${ }^{43}$.

On peut ajouter ici la place que représente l'expression «association politique» pour désigner le Brésil ${ }^{44}$ dans la traduction : il est traduit par le terme danketsu 団結 («union»), qui n'est, à notre connaissance, guère utilisé à l'époque. Or non seulement il était un terme favori de Chômin, qui contribuera à relancer le Mouvement pour les libertés et les droits du peuple en 1886-7 sous le nom d'Union des groupes partageant les mêmes buts (Daidô danketsu 大同団結) mais l'association politique est une évocation indirecte de la théorie contractualiste. En d'autres termes, le terme illustrait un lien étroit entre la traduction du Contrat social et celle de la Constitution brésilienne: il s'agissait dans les deux cas d'affirmer que le Japon pouvait être un corps politique constitué par la volonté libre des citoyens et non par celle du monarque (ou des oligarques).

La présence du terme «association politique» n'est pas dénué d'ironie: comme Emilia Viotti Da Costa l'indique, l'article un de la constitution où figure cette expression est une reprise du début de la Déclaration de $1789^{45}$. Chômin faisait grand cas de la Révolution française et traduisit, entre autres, la constitution de 1793. Chômin a peutêtre remarqué cette ressemblance mais il n'aura pas compris que la version brésilienne tronquait largement l'article de la Déclaration puisque l'affirmation des droits naturels

42 Repris de VERMOREL Auguste, Mirabeau. Sa vie, ses opinions et ses discours, Paris, Librairie de la bibliothèque nationale, 1880, pp.126-139; «Sensei seiji no shukuhei wo ronzu» 専制政治ノ宿弊习論 ズ (Sur les calamités du gouvernement despotique), Ôbei seiri sôdan, 1, 3, 4, 5, 6.

43 BENTHAM Jeremy, De l'organisation judiciaire et de la codification, Paris, H.Bossange, 1828, p.13; Ôbei seiri sôdan, 9, p.125.

44 LAFERRIÈRE, op.cit., p.589.

45 VIOTTI DA COSTA, Emilia, Da Monarquia à republica, São Paulo, Fundação Editora da Editora Unesp, 1998, p.144. Article 2 de la Déclaration de 1789: «Le but de toute association politique est la conservation des droits naturels et imprescriptibles de l'Homme. Ces droits sont la liberté, la propriété, la sûreté, et la résistance à l'oppression». 
y était complètement absente. L'article 3 de la Déclaration affirmant la souveraineté de la nation n'est pas davantage repris dans la constitution brésilienne. Or, justement, il est remarquable que la traduction japonaise transforme le texte originel de l'article pour affirmer les droits du peuple et opérer ainsi un retour à l'article de 1789. Mettre côte à côte les deux versions fait ressortir cette différence :

\section{Version française (à partir du portugais)}

«L'empire du Brésil est l'association politique de tous les citoyens brésiliens. Il forme une nation libre et indépendante, qui n'admet avec aucune autre lien d'union ou de fédération qui puisse porter atteinte à son indépendance. »

\section{Version japonaise (à partir du français) ${ }^{46}$}

巴西国帝国八巴西国民ノ其政习為スノ便ヨ計ランカ為メニ団結スル者ナリ而 シテ其国タルヤ不羈独立ニシテ其民人タルヤ自由権アリ既ニ自由権アリ然レドモ 国家ノ独立习損傷スルカ如キ他邦連合ハ之ヨ盟約スルコト許サス

«L'empire du Brésil est formé de l'union des citoyens brésiliens en vue de constituer son gouvernement. Ce pays est indépendant et son peuple jouit du droit à la liberté. Ce droit à la liberté existe mais il ne sera pas toléré d'union avec d'autres pays qui pourrait nuire à l'indépendance de la nation».

Le plus étonnant est que la suite de la traduction est très fidèle à l'originale, de sorte qu'il ne fait aucune doute d'une part que la constitution donne au roi un pouvoir prépondérant et d'autre part qu'elle reconnaît une oligarchie puisque les membres de la chambre haute sont en partie nommés par le souverain et, comme ceux de la chambre basse, assez riches pour payer un cens élevé. Il y a donc clairement une contradiction dans la traduction des articles 1 et 99 , qui n'hésite pas à transformer le texte original au sens désiré et le reste de la traduction, qui rend claire l'orientation autoritaire de la constitution brésilienne. Chômin est-il intervenu au tout début de la traduction pour ensuite la laisser au traducteur? Le mystère reste entier.

On le relèvera à cet égard que la Constitution brésilienne de 1824 est souvent présentée comme un produite des idées de Benjamin Constant (Principes politiques) et de reprendre de lui la séparation en quatre pouvoirs, distinguant un pouvoir modérateur au-dessus des trois autres ${ }^{47}$. Propre au roi, il permettait chez Constant l'harmonie entre

46 Graphie modernisée pour les idéogrammes. Le terme kokumin qui apparaît ici traduit aujourd'hui le mot «nation» mais ici il est systématiquement utilisé pour traduire «citoyen».

47 Constant n'était pas la seule source d'inspiration. Il faut ajouter le juriste Jean-Denis Lanjuinais (17531827), lui-même lecteur de Bernardin de Saint-Pierre et d'autres publicistes moins connus, qui eurent un rôle plus important que Constant lui-même dans l'élaboration de la Constitution de 1824. Voir FERREIRA, Oscar, «Le pouvoir modérateur dans la Constitution brésilienne de 1824 et la Charte 
les trois autres. Dans la Constitution brésilienne ce pouvoir est dévolu à l'empereur, lui permettant de nommer et démettre les ministres, nommer les sénateurs et dissoudre la chambre. Si Chômin n'a jamais évoqué le pouvoir modérateur dans ses propres écrits, il fit traduire le fameux article de Constant où celui-ci reproche à la volonté générale rousseauiste d'être une tyrannie de la majorité, critique reprise par d'autres auteurs traduits par Chômin et ses élèves (Jules Barni et Sigismond Lacroix) ${ }^{48}$. Ainsi, Rousseau est présent de manière implicite dans la traduction de la Constitution brésilienne et cela constitue peut-être une raison supplémentaire de son choix.

Néanmoins il semblerait que la présence de Constant dans la Constitution de 1824 soit un mythe et que l'inspiration libérale était dévoyée notamment en raison de l'article 102 qui pose une frontière floue entre le pouvoir exécutif et le pouvoir modérateur, permet au souverain d'incarner un pouvoir conservateur et d'interférer dans la vie privée des individus sur le plan religieux, autant de points que Constant n'aurait pas acceptés ${ }^{49}$. La traduction japonaise étant fidèle, il semble que son auteur ne se soit pas aperçu de ce problème, qui nuance fortement l'apparente suprématie du pouvoir législatif dans la constitution au profit du pouvoir modérateur et exécutif, c'est-à-dire l'empereur. C'est précisément qui a permis aux conservateurs d'interpréter la constitution dans un sens autoritaire (jusqu'à l'amendement de 1846), ce que ne semble pas avoir su les traducteurs. Trop dépendant de Laferrière, Chômin et le traducteur d'Ôbei seiri sôdan auraient introduit ainsi un texte allant aux antipodes de leurs buts politiques.

Néanmoins, certaines dispositions de la constitution donnent au gouvernement une certaine responsabilité envers les citoyens, ce qui a sans doute plu à Chômin et à ses élèves, désireux, comme tous les partisans du Mouvement pour les libertés et les droits du peuple, de lutter contre l'autoritarisme de leur gouvernement. Par exemple, aux sujets des ministres, l'article 133 indique clairement qu'ils sont responsables pour trahison, pour corruption, subordination ou concussion, pour abus de pouvoir, pour défaut d'observation de la loi, pour utilisation de l'argent public et surtout «pour ce qu'ils feraient contre la liberté, la sécurité ou la propriété des citoyens $»^{50}$. Les articles 134 et 135 précisent même qu'une loi particulière précisera la procédure à leur égard et que l'empereur ne pourra agir en faveur des ministres coupables. Tout cela est fidèlement

constitutionnelle portugaise de 1826: les influences de Benjamin Constant ou de Lanjuinais?», Revue française de droit constitutionnel, 89, janvier 2012, pp.1-40.

48 CONSTANT, Benjamin, «De la souveraineté du peuple», dans LOUANDRE, Charles ed., CEuvres politiques de Benjamin Constant, Paris, Charpentier et Cie, 1874, Id., «Shuken zaiminron» 主権在民 論 (De la souveraineté du peuple), Ôbei seiten shûshi, 15, 1886, pp. 26-7. LACROIX, Sigismond, «De la souveraineté du peuple», La Science politique, 5, 1878, pp. 37-41. «Shuken zaiminron» 主権 在民論 (De la souveraineté du peuple), Ôbei seiri sôdan, 31, 1883, et «Hi Shuken zaimin ron» 非主権 在民論 (De la non-souveraineté du peuple), Ôbei seiten shûshi 欧米政典集誌, 9, 1886, pp. 1-4; 12, pp. 10-21.

49 FERREIRA, op.cit., pp.15-6.

50 LAFERRIÈRE, op.cit., p.609; Ôbei seiri sôdan, 53, p.1880. 
traduit en japonais. À une époque où les partisans du Mouvement remettaient en cause la toute-puissance des ministres-oligarques et avaient profité d'une affaire de corruption touchant une partie du gouvernement pour critiquer l'ensemble des dirigeants, de telles dispositions de la Constitution brésilienne ne pouvaient sembler que progressistes.

Enfin, les articles concernant la défense ne sont pas sans intérêts. Selon l'article 145, «tous les Brésiliens sont tenus de prendre les armes pour conserver l'indépendance et l'intégrité de l'empire, et pour le défendre contre les ennemis du dedans et du dehors» ${ }^{51}$. Même si les articles suivant indiquent que des armées permanentes seront établies à terme, cet article 145 qui décrit l'usage militaire en vue de défense, par le peuple en armes, n'est pas sans rappeler le pacifisme de Chômin. En effet, celui-ci est bien connu pour avoir adossé sa critique de l'impérialisme de son gouvernement par une proposition de constituer des forces militaires non permanentes à partir du peuple en armes, en vue de l'auto-défense ${ }^{52}$. Dans les Dialogues politiques entre trois ivrognes, Chômin fait exprimer cette opinion par Maître Nankai et place dans la bouche de Mr Le Gentleman la traduction de Jules Barni, expliquant les thèses de Bernardin de Saint Pierre et de Kant au sujet de la paix perpétuelle ${ }^{53}$. Jules Barni était l'organisateur, avec Emile Acollas, du Congrès de la paix de Genève en 1869. Cette affirmation de la thèse d'armée non permanentes, que défendra Jean Jaurès, n'a pas qu'une dimension internationale mais est liée aussi à la volonté de démocratiser le Japon: on peut y voir un moyen stratégique pour combattre la violence d'Etat que le gouvernement usait constamment tout au long des années 1880 contre le Mouvement pour les libertés et les droits du peuple (et Chômin lui-même). Dans un pays débarrassé des armées permanentes et où le peuple détiendrait les armes, le gouvernement aurait été bien incapable d'utiliser des lois, la police ou l'armée contre le Mouvement pour les libertés et les droits du peuple. Surtout, Chômin a exprimé son pacifisme surtout dans la deuxième moitié des années 1880. Ainsi la traduction de l'article 145 de la Constitution brésilienne préfigurait ce pacifisme et l'a sans doute inspiré, comme plusieurs textes francophones traduits dans Ôbei seiri sôdan et ailleurs ${ }^{54}$.

En ce qui concerne le corps de la traduction, il est remarquable qu'elle est dans sa globalité fidèle à l'original. Ainsi les quelques libertés que le traducteur a prises n'en sont que plus manifestes. Tout d'abord, si l'article 5 faisant du christianisme la religion

51 LAFERRIÈRE, op.cit., p.609; Ôbei seiri sôdan, 53, p.1881.

52 NAKAE, Chômin, «Dochakuheiron»土着兵論 (Sur l'armée du peuple; 16 mai 1888), NCZ, 11, pp.142-152.

53 NAKAE, Chômin, Dialogues politiques entre trois ivrognes, op.cit., pp.89-90, 135-140. Chômin évoque également Bernardin de Saint-Pierre et Kant dans NAKAE, Chômin, «Zenkoku shinbun zasshi narabi choyakusho hihyô (yon)» 全国新聞雑誌並著訳書批評 (Evaluation des journaux et des revues nationales ainsi que des essais et traductions, 4; 15 février 1889), NCZ, 15, p.21.

54 Par exemple VACHEROT, Etienne, La Démocratie, Paris, 1860. Voir DUFOURMONT, Eddy, «Un cas d'étude de transfert culturel au Japon: Nakae Chômin (1847-1901), Rousseau et le républicanisme français», op.cit., pp.456-472. 
d'Etat est fidèlement traduit, tout le préambule écrit «au nom de la sainte trinité» manque. Comme Chômin était un athée convaincu, dont le livre de chevet était celui d'Emile Accolas, Philosophie des sciences politiques et commentaire de la déclaration de 1793 (1877), tout entier tourné contre les religions ${ }^{55}$. Chômin était d'ailleurs très intéressé par le positivisme ${ }^{56}$ et la présence forte de celui-ci au Brésil a peut-être attiré le regard de Chômin. Ceci reste néanmoins une pure supposition puisque Chômin n'établit jamais lui-même ce lien. Par ailleurs, la constitution reconnaît une certaine liberté de croyance. Celle-ci n'étant pas encore établie au Japon (l'interdiction du christianisme fut simplement levée en 1873), la traduction pouvait contribuer à l'affirmation de cette liberté.

\section{Conclusion}

Au final, en faisant traduire la Constitution brésilienne de 1824, Chômin et ses élèves semblent avoir voulu donner aux lecteurs un exemple de constitution de monarchie constitutionnelle, au même titre que la Grande-Bretagne et la Belgique. Cette constitution, mêlant libéralisme et absolutisme, fut clairement interprétée et détournée dans un sens libéral par la traduction, avec quelques maladresses. Elle montre néanmoins que la recherche d'un modèle politique chez les libéraux japonais tels que Chômin embrassait la terre entière et était loin de se limiter à la France ou l'Angleterre. Un an après Chômin et ses élèves, le gouvernement utilisa lui aussi l'ouvrage de Laferrière pour publier un panorama des constitutions, limité aux chambres hautes. Contrairement à Ôbei seiri sôdan, le Brésil y occupe une place minime, en annexe du Portugal ${ }^{57}$. Peutêtre faut-il y voir une preuve que la Constitution de 1824, malgré la présence du pouvoir modérateur, apparaissait bien aux yeux des libéraux japonais comme un modèle possible et pour le gouvernement des oligarques comme une menace.

55 Voir ACOLLAS, Emile, Philosophie de la science politique et commentaire de la déclaration des droits de l'homme de 1793, Paris, A.Marescq aîné, 1877.

56 À ce sujet voir MIYAMURA Haruo宮村治雄, Rigakusha Chômin 理学者兆民 (Chômin philosophe), Tôkyô, Perikansha ペリカン社 1986, pp.7-141; DUFOURMONT, Eddy, «Un cas d'étude de transfert culturel au Japon: Nakae Chômin (1847-1901), Rousseau et le républicanisme français», op.cit., pp.15, 233.

57 Kakoku jôin kiyô各国上院紀要 (Précis des chambres hautes des pays), Tôkyô, Genrô.in 元老院, 1884, p.193. 